\title{
Properties of Dusty Plasma Crystal Accounting Charge Variety of Dust Particles
}

\author{
Konstantin P. Zolnikov ${ }^{1,2, a)}$, Andrei V. Abdrashitov ${ }^{1}$, \\ Dmitrij S. Kryzhevich ${ }^{1,2}$, and Sergey G. Psakhie ${ }^{1,3,4}$ \\ ${ }^{1}$ Institute of Strength Physics and Materials Science SB RAS, Tomsk, 634055, Russia \\ ${ }^{2}$ National Research Tomsk State University, Tomsk, 634050, Russia \\ ${ }^{3}$ National Research Tomsk Polytechnic University, Tomsk, 634050, Russia \\ ${ }^{4}$ Skolkovo Institute of Science and Technology, Skolkovo, 143025, Russia

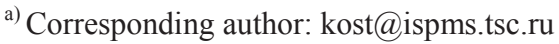

\begin{abstract}
Shock wave properties generated by impulse mechanical loading and base state structure of dusty plasma system versus the change of dusty particle charges with height were investigated in the framework of the molecular dynamics method. Spherically symmetric electrostatic confinement was used as the boundary conditions. Interactions between dusty particles were described using Debye-Huckel potential. Particle charges decreased linearly with the increase of height. Shape of dusty plasma crystals with taking into account a variable particle charge was simulated.
\end{abstract}

Keywords: molecular dynamics, dusty plasma

\section{INTRODUCTION}

Dusty or complex plasma has been investigated with great attention during the last decades. It represents an ionized gas where the charged particles of condensed matter with microscopic sizes are placed. An electric charge of dust particles may reach several thousand electrons therefor they interact among themselves strongly with Coulomb forces. It should be noted that dusty plasma systems are widely spread in space and laboratory conditions, particularly in a number of processes connected with ion-plasma treatment of material surface. The variability of the microparticles charge and external impulse effects of different nature leads to generating in dusty plasma specific wave modes. The study of wave excitations in dusty plasma systems, dynamics of their propagations, peculiarities of the interaction with each other, the nature of their attenuation, various kinds of instabilities is an important scientific direction in the field of dusty plasma [1]. For the first time the dust-acoustic solitary waves in magnetized dusty plasma were studied in [2]. In the subsequent paper [3] the possibility of soliton and shock wave propagation in dusty plasma was shown. The linear acoustic waves were obtained experimentally in [4], and in [5] Mach cones generated by the motion of the object through the medium at supersonic speed were observed.

In the present work, the characteristics of the shock waves generated in dusty plasma systems under impulse mechanical loading were studied within the framework of computer modeling. To solve this problem, we used the method of molecular dynamics. The object of the study was the extended one-component dusty plasma system in near base (crystalline) state, located in the cylindrical confining field of electrostatic nature. Absolutely hard walls along the axis of the cylinder at its edges were used as the boundary conditions in order to hold charged dust particles from expansion.

\section{FORMALISM}

The interaction between dust particles was determined by the potential of the Debye-Huckel:

$$
\varphi(r)=\frac{Q}{r} \exp \left(-\frac{r}{\lambda_{\mathrm{D}}}\right),
$$

International Conference on Physical Mesomechanics of Multilevel Systems 2014

AIP Conf. Proc. 1623, 671-674 (2014); doi: 10.1063/1.4899034

(C) 2014 AIP Publishing LLC 978-0-7354-1260-6/\$30.00 

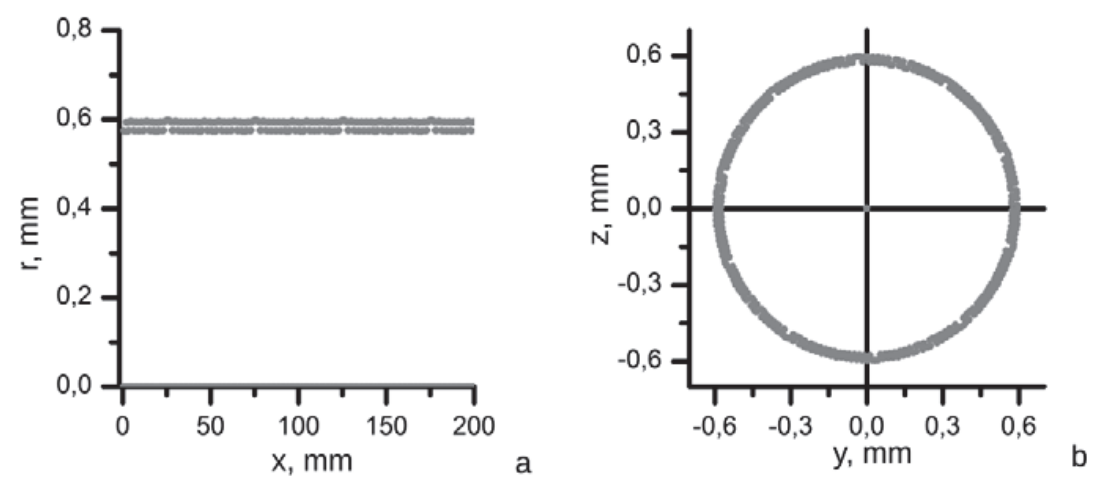

FIGURE 1. Structure projection of the simulated dusty plasma system on the plane:

(a) $x-r$, where $r$ is the distance from the axis of the cylinder to the particle; (b) $y-z$

where $\lambda_{\mathrm{D}}$ - the screening length plasma (Debye radius), $Q$ - the charge of a dust particle. The force acting from the trap on dust particles and keeping them from expansion had electrostatic nature and was described by the expression:

$$
F=-\alpha Q(y+z),
$$

where $\alpha$-the factor determining the confinement field force in the direction perpendicular to the axis of the cylinder (in our calculation $\alpha=10^{-14}$ ). Dust particles have a spherical shape, their density corresponds to melamine formaldehyde, their radius is equal to $3.585 \mu \mathrm{m}$, and the charge is $2660 \mathrm{e}$. The simulated dusty plasma system contained 1684 particles. The calculations showed that the interaction between dust particles becomes negligibly small in the distance more than $3 \mathrm{~mm}$. To reduce the calculation time, this distance was made for the cutoff radius in the interaction potential.

For the above boundary conditions, the investigated dusty plasma system had the shape of a cylinder. Being in crystalline state, this system took a shell structure. Its projections are presented in Fig. 1. The figure shows that only one shell was formed in the investigated system. The dust particles were located either along the axis of the cylinder, or along a shell at the distance of $0.6 \mathrm{~mm}$ from the axis.

Loading of the simulated system was carried out in the direction of the axis of the cylinder. To initiate the waves of one edge of the cylinder, capture (its size along the axis was equal to two of the cutoff radius) was moved at a constant speed of $10 \mathrm{~mm} / \mathrm{s}$ at a distance: $0.01 \mathrm{~mm}$, to $0.1 \mathrm{~mm}$ and $1 \mathrm{~mm}$ in different calculations. After the cylinder position displacement the capture was fixed, and dust particles in it were stopped too.

The generated perturbations propagated in the simulated system along the $X$-axis (the axis of the cylinder). Note that the generation of the perturbations (waves) was specified in two ways: shock compression or tension of dusty plasma system.
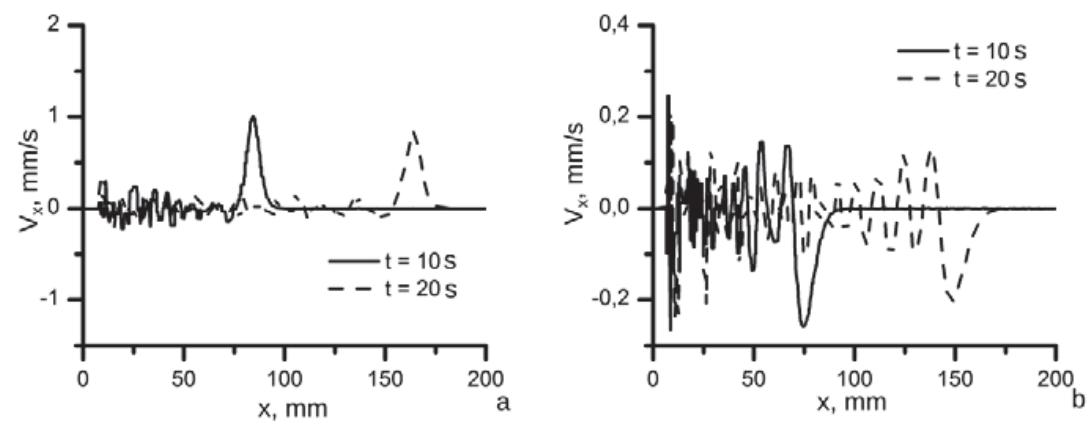

FIGURE 2. The velocity distribution of dust particles along the direction of loading after the displacement of left capture by $1 \mathrm{~mm}$ with a constant speed $10 \mathrm{~mm} / \mathrm{s}$ at different points in time: compression (a) and tensile (b) 

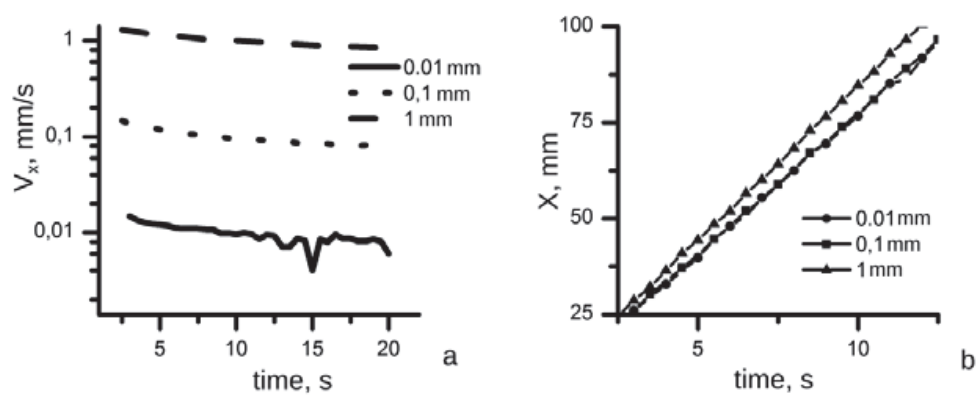

FIGURE 3. The dependence of the amplitude of the first compression wave $V_{x}$ (a) and its location $X$ (b) on time

\section{COMPUTATION RESULTS}

The results of the calculations showed that perturbations formed in the study of dusty plasma system were the nonlinear waves of compression and tensile. The velocity distribution of dust particles in the direction of the $X$ axis at different points in time for the two-ways pulse effect is shown in Fig. 2. The nonlinear compression wave propagated in dusty plasma system ahead of perturbation after the compression displacement of the capture (Fig. 2(a)), and the tensile wave propagated ahead after the tensile displacement of the capture (Fig. 2(b)). The analysis of the simulation results showed that the wavelength increased during its propagation. The amplitude of the first wave, which had a maximum value, decreased exponentially as it propagated (Fig. 3(a)). Sufficiently quick decreasing the amplitude of this wave was connected with its interaction with other waves generated by the capture motion. It should be noted that the propagation of the nonlinear waves did not lead to the violation of the shell structure. Within the simulated dusty plasma system, the speed of wave propagation remained approximately constant (Fig. 3(b)).

The analysis of the simulation results shows that the velocity of the nonlinear wave propagation depends on the wave amplitude. For the case, when the simulated system is compressed, the data for calculating the amplitude (after $2.5 \mathrm{~s}$ from the moment of generation), the velocity of the compression wave propagation, and the relative density of dust particles in a wave are presented in the table. It is supposed that in the initial system before loading the density of particles was equal to 1 . The Table 1 clearly shows that the increase in the dust particles density in the nonlinear wave leads to greater velocity of its propagation.

The results of calculations are in a qualitative agreement with the available experimental data [6]. The experiments on the generation of waves in dusty-plasma structure in the DC discharge have been carried out and the peculiarities of propagation of compression and rarefaction waves have been studied in this work.

For a more realistic description of the dusty plasma crystals structure, it is necessary to take into account the charge variability of dust particles with height. In this work, the variation in particle charge was set by the following expression:

$$
Q=Q_{0}(1+\mathrm{d} Q / \mathrm{d} z)
$$

The value of $\mathrm{d} Q / \mathrm{d} z$ determined the rate of charge change with height. The charge $Q_{0}$ for all particles was equal to 2660e. The trap force acting on dust particles and keeping them from expansion had electrostatic nature and was described by the expression:

TABLE 1. Dependence of the compression wave propagation velocity and its characteristics on the value of loading

\begin{tabular}{cccc}
\hline $\begin{array}{c}\text { Value of Loading, } \\
\mathbf{m m}\end{array}$ & $\begin{array}{c}\text { Amplitude of Compression } \\
\text { Wave, } \mathbf{m m} / \mathbf{s}\end{array}$ & $\begin{array}{c}\text { The Wave } \\
\text { Propagation Velocity, } \\
\mathbf{m m} / \mathbf{s}\end{array}$ & $\begin{array}{c}\text { Relative Density of Dust } \\
\text { Particles in the Region } \\
\text { of Wave }\end{array}$ \\
\hline 0.01 & 0.015 & 7.3 & 1.02 \\
\hline 0.10 & 0.150 & 7.4 & 1.07 \\
\hline 1.00 & 1.300 & 8.0 & 1.12 \\
\hline
\end{tabular}




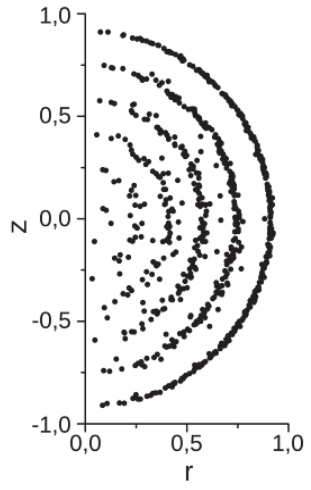

(a)

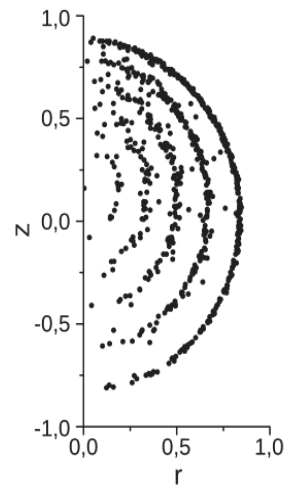

(b)

FIGURE 4. Structure projection of dusty plasma system in dimensionless cylindrical coordinates. Simulated dusty plasma crystal contains 720 particles: (a) $\mathrm{d} Q / \mathrm{d} z=0$, (b) $\mathrm{d} Q / \mathrm{d} z=1000$

$$
F=-\alpha Q(r+z),
$$

where $\alpha$-the factor determining the force of confinement field (in the calculations of $\alpha=10^{-14}$ ), $r$ is the distance from the cylinder axis to a particle, and $z$-height. If the rate of the charge change is equal to 0 , then dusty plasma system forms the so-called Coulomb ball (Fig. 4(a)) having clearly seen shell structure [7, 8]. The simulated plasma crystal has a more complex structure if a charge change of the dust particles with height is not equal to 0 (Fig. 4(b)).

The work was partially supported by the program of basic research with the Presidium of the Russian Academy of Sciences No. 2 "Matter at high energy densities" and program for competitiveness enhancement of Tomsk State University.

\section{REFERENCES}

1. V. E. Fortov, A. G. Khrapak, S. A. Khrapak, V. I. Molotkov, and O. F. Petrov, Physics-Uspekhi 47, 447 (2004).

2. N. N. Rao, P. K. Shukla, and M. Y. Yu, Dusty Plasmas 38, 543 (1990).

3. P. K. Shukla and A. A. Mamun, Introduction to Dusty Plasma Physics (IOP, Bristol, 2002).

4. A. Barkan, R. L. Merlino, and N. D’Angelo, Phys. Plasmas 2, 3563 (1995).

5. D. Samsonov, J. Goree, Z. W. Ma, A. Bhattacharjee, H. M. Thomas, and G. E. Morfill, Phys. Rev. Lett. 83, 3649 (1999).

6. V. E. Fortov, O. F. Petrov, V. I. Molotkov, M. Y. Poustylnik, V. M. Torchinsky, A. G. Khrapak, and A. V. Chernyshev, Phys. Rev. E 69, 016402 (2004).

7. S. G. Psakhie, K. P. Zolnikov, L. F. Skorentsev, D. S. Kryzhevich, and A. V. Abdrashitov, Phys. Plasmas 15, 053701 (2008).

8. S. G. Psakhie, K. P. Zol'nikov, L. F. Skorentsev, D. S. Kryzhevich, and A. V. Abdrashitov, Tech. Phys. Lett. 34, 319 (2008). 